Cite this: J. Mater. Chem. C, 2014, 2 , 1742

Received 18th September 2013 Accepted 15th December 2013

DOI: $10.1039 / c 3 t c 31849 d$

www.rsc.org/MaterialsC

\title{
Characterisation of charge conduction networks in poly(3-hexylthiophene)/polystyrene blends using noise spectroscopy $\dagger$
}

\begin{abstract}
Aled T. Williams, ${ }^{a}$ Paul Farrar, ${ }^{a}$ Andrew J. Gallant, ${ }^{a}$ Del Atkinson ${ }^{b}$ and Chris Groves ${ }^{\star a}$
$1 / f$ noise spectroscopy is used to investigate charge conduction networks within polymer blend spacecharge-limited diodes (SCLDs) fabricated from regioregular poly(3-hexylthiophene) (P3HT) and either isotactic-polystyrene (i-PS) or amorphous-polystyrene (a-PS). Conducting AFM measurements showed that i-PS blends have heterogeneous conduction characterised by current 'hotspots', whereas a-PS blends showed homogeneous conduction. The difference in conducting networks between blends was clearly revealed when examining the noise spectra for the range of blend devices. Furthermore, the shape of the noise spectra suggested that as the blend composition changed, the charges sampled differing breadths of the density of states. These data suggest that noise measurements can be used as an informative technique to electrically characterise the effects of blend morphology and its effects within polymer electronic devices.
\end{abstract}

\section{Introduction}

An advantage of solution-processable organic electronic devices is the ability to blend different materials to endow the active layer with properties that would be impossible with one material alone. Compelling examples of this include bulk heterojunction organic photovoltaic devices (OPVs), in which efficient charge generation is enabled by a nanometre-scale network of materials with different electron affinities, ${ }^{1,2}$ and white organic light-emitting diodes (WOLEDs), which use blends to optimise charge transport and achieve the correct colour balance., ${ }^{3,4}$ Blending has benefits that extend beyond enhanced electrical and optical properties, since insulating polymers can also be used to modify mechanical ${ }^{5}$ and degradation ${ }^{6}$ properties of the active layer. Whatever the intended benefits, a common consequence of blending is that it imposes a 3-dimensional network through which charges are injected, extracted, and otherwise move or recombine. Optimising the charge percolation network and its associated properties ${ }^{7-9}$ is often a key part of optimising device performance.

Solution processing can result in a variety of morphologies, and therefore charge percolation networks, depending upon the materials and deposition methods used. ${ }^{\mathbf{8} 10-12}$ In turn, this diverse range of charge percolation networks, hereafter networks, can lead to a range of electrical properties that can be

${ }^{a}$ School of Engineering and Computing Sciences, Durham University, Durham, DH1 3LE, UK. E-mail: chris.groves@durham.ac.uk

${ }^{b}$ Department of Physics, Durham University, Durham, DH1 3LE, UK

$\dagger$ Electronic supplementary information (ESI) available. See DOI: $10.1039 / \mathrm{c} 3$ tc31849d hard to predict. ${ }^{12}$ Informative experimental techniques showing the network, and the consequences on its electronic performance, are therefore necessary to obtain greater understanding of how organic electronic devices operate. Fortunately, there are many techniques available to probe the nature of the bulk and surface morphology in blended organic electronic devices. ${ }^{13,14}$ However, there are arguably fewer experimental techniques which are used to examine the detail of the electronic performance, and in particular, to relate this electrical performance to a given morphological network.

In this paper, we use noise spectroscopy as a tool for in situ characterisation of networks within blended organic electronic devices. Noise is the fluctuation of voltage across or current through an electronic device, which is a result of random processes occurring during device operation, and is characterised by the power spectral density, $S_{I}(f)$. Of the types of intrinsic electronic noise, we here focus on $1 / f$, or 'flicker', noise ${ }^{15-18}$ which can dominate $S_{I}(f)$ at low frequencies (typically $<1 \mathrm{kHz}) .1 / f$ noise is usually the result of conductivity fluctuations within a material ${ }^{17-19}$ and so can reveal information about dynamic processes such as trapping and detrapping. ${ }^{20}$ While noise spectroscopy has been used extensively to understand trap-mediated charge transport within inorganic electronic devices, ${ }^{20}$ rather fewer investigations have been reported for organic electronic devices. Notable exceptions include the use of noise spectroscopy to better understand trapping in organic field effect transistors (OFETs), ${ }^{21-25}$ and to examine the relationship between trap formation and device lifetime in OLEDs ${ }^{26}$ and OPVs. ${ }^{16}$

However, here we utilise another property of noise spectroscopy, namely that $1 / f$ noise is sensitive to the nature of the 
conducting network. In a blend with a conducting and nonconducting component, the properties of the network can be described by the volume fraction of conducting material, $p$. Below a threshold value of $p$ the conductivity of the network will tend toward zero, known as the percolation threshold, $p_{\mathrm{c} \cdot}{ }^{18}$ In this situation, the $1 / f$ noise power spectral density close to the percolation threshold scales as follows:

$$
\left(p-p_{\mathrm{c}}\right)^{-\kappa}
$$

Here $\kappa$ is the percolation exponent of noise which is dependent on the dimensionality of the system. ${ }^{27}$ The relationship between $1 / f$ noise and $p$ therefore reveals information about the percolation threshold and the nature of the conducting network within the device. This relationship has already been used to examine percolation in pentacene OFETs fabricated by evaporation, in which grain boundaries pose a barrier to conduction. ${ }^{28}$ However, to the best of our knowledge, the relationship between $1 / f$ noise and the charge conduction network in organic blended devices has not been examined.

Here we report investigations on a range of space-chargelimited diodes (SCLDs) using conductive regioregular poly(3hexylthiophene) (P3HT) and insulating polystyrene (PS). The device morphology is controlled via the blend ratio and by use of either isotactic-PS (i-PS) or atactic-PS (a-PS). Conductive AFM (c-AFM) measurements showed that i-PS and a-PS blends had heterogeneous and homogeneous conduction pathways through the film respectively; in agreement with previous investigations. ${ }^{\mathbf{6}, 12}$ Noise spectroscopy measurements were then carried out on the same devices. These data confirmed the validity of eqn (1) in blended organic electronic devices, with analysis of $\kappa$ suggesting a network in qualitative agreement with that found by c-AFM measurements. Consequently, noise spectra can differentiate between SCLDs with heterogeneous and homogeneous networks, even when they had similar average current levels. The data also appear to show that the shape of the noise spectrum is sensitive to the density of states (DoS) sampled by charges en-route through the film. These findings show that noise spectroscopy is a useful additional tool to understand how electrical performance and morphology relate in organic electronic devices.

\section{Experimental}

\section{Materials}

Regioregular P3HT (electronic grade; $M_{\mathrm{w}}=50-70 \mathrm{~kg} \mathrm{~mol}^{-1}$ ) was supplied by Rieke Metals, Inc. and used as received. i-PS $\left(M_{\mathrm{w}}=\right.$ $\left.400 \mathrm{~kg} \mathrm{~mol}{ }^{-1}\right)$, a-PS $\left(M_{\mathrm{w}}=400 \mathrm{~kg} \mathrm{~mol}^{-1}\right)$ and the solvent anhydrous 1,2,4-trichlorobenzene (TCB) were purchased from Sigma-Aldrich and used as received. The poly(3,4-ethylenedioxythiophene):poly(styrenesulfonate) (PEDOT:PSS) was purchased from Clevios, kept refrigerated and shaken before use.

\section{Device fabrication}

ITO bottom electrodes were patterned into strips via Zn powder assisted HCl-etching of ITO-coated glass slides. The substrates were then cleaned using the following procedure: rinsed in propan-2-ol; 15 min sonication in propan-2-ol; rinsed in propan-2-ol; dried in $\mathrm{N}_{2}$ gas; rinsed in acetone; 15 min sonication in acetone; rinsed in acetone; dried in $\mathrm{N}_{2}$ gas; rinsed in ultrapure water; 15 min sonication in Decon 90 solution (2\% aqueous); rinsed in ultrapure water; 15 min sonication in ultrapure water; dried in $\mathrm{N}_{2}$ gas. Substrates were exposed to $\mathrm{O}_{2}$ plasma (100 W, $5 \mathrm{~min}$ ) and within $1 \mathrm{~min}$ a $c a .45 \mathrm{~nm}$ layer of (PEDOT:PSS) was spin-coated (2500 rpm for $45 \mathrm{~s}$ ) onto the ITO electrode, followed by annealing at $180{ }^{\circ} \mathrm{C}$ for $2 \mathrm{~min}$. Active layer blends were prepared by dissolving the materials separately in TCB to produce $1 \mathrm{wt} \%$ solutions, these were then mixed to desired P3HT:PS ratios and stirred for a $24 \mathrm{~h}$ period. Blends were deposited onto substrates via spin-coating (1000 rpm for $60 \mathrm{~s}$ ), with both the solutions and substrates held at $120{ }^{\circ} \mathrm{C}$ (above the glass-transition temperature of PS in order for the P3HT to crystallise before the solidification of the PS matrix ${ }^{\mathbf{1 2}}$ ), to produce $c a .40 \mathrm{~nm}$ thick films; all performed within a $\mathrm{N}_{2}$ filled glove box. Devices were then annealed at $120^{\circ} \mathrm{C}$ for $20 \mathrm{~min}$ to promote further crystallisation before $\mathrm{Au}$ top electrodes were deposited via masked evaporation to a thickness of $150 \mathrm{~nm}$ and with a cross-sectional area of $3.53 \times 10^{-6} \mathrm{~m}^{2}$. The volume ratios of the components in the film were calculated from the relative weight percentage of the components in solution and the respective densities: P3HT $\left(1.10 \mathrm{~g} \mathrm{~cm}^{-3}\right){ }^{29}$ a-PS $\left(1.05 \mathrm{~g} \mathrm{~cm}^{-3}\right)^{30}$ and i-PS $\left(1.12 \mathrm{~g} \mathrm{~cm}^{-3}\right) .{ }^{31}$

\section{I- $V$ characterisation}

Current-voltage measurements were performed in the dark and under vacuum using a Source Measure Unit (Keithley 2400).

\section{Noise spectroscopy}

Noise measurements were carried out at $293 \mathrm{~K}$ and at a current of $3 \mathrm{~mA}$ under vacuum and in the dark, with all parts of the measurement rig being electrically screened. Current fluctuations were amplified using a low-noise current pre-amplifier (Stanford Research, SR570) and the analog signal was sampled using a 24-bit data acquisition card (DAQ) (National Instruments, NI USB-4431). The data was processed using a custombuilt LabVIEW program to produce the noise spectrum.

\section{AFM and c-AFM measurements}

All AFM measurements were performed in a clean room environment at $20 \pm 2{ }^{\circ} \mathrm{C}$ and relative humidity of $45 \pm 10 \%$ using a Dimension 3100 microscope and Nanoscope IVa controller (Bruker). c-AFM measurements were obtained in contact mode using $\mathrm{Au}$ coated AFM probes with a $\mathrm{Cr}$ adhesion layer (Budget Sensors ContGB-G, nominal spring constant $=0.2 \mathrm{~N} \mathrm{~m}^{-1}$ ) as the top contact on the device. Electrical current at the tip was measured in dark conditions under a forward bias of $1 \mathrm{~V}$, using a current amplifier (Bruker Extended Tunnelling Current module) attached to the AFM. In this set up the tip is held at virtual ground and bias applied to the ITO via a silver paint contact to the AFM stage. AFM and c-AFM measurements were taken simultaneously on the same area of film, and on a number of different areas across the film surface to ensure that the observed features were typical. Film thickness 
measurements were obtained using the same AFM operated in dynamic mode with uncoated silicon nitride AFM probes (Budget Sensors Tap300Al-G, nominal spring constant = $40 \mathrm{~N} \mathrm{~m}^{-1}$ ).

\section{UV-Visible spectroscopy}

Films were measured using a spectrophotometer (Shimadzu UV-1800). The P3HT $\lambda_{\max }$ was found to be at $511 \mathrm{~nm}$.

\section{Results and discussion}

\section{P3HT:a-PS blend characteristics: isotropic networks}

Hole-only ITO/PEDOT:PSS/P3HT/Au SCLD diodes were fabricated and compared to blended ITO/PEDOT:PSS/a-PS:P3HT/Au devices in which the a-PS is used to manipulate the conductive P3HT networks throughout the film. As in the terminology of eqn (1), the volume percentage of conducting P3HT in the film is referred to as $p$, while the insulating a-PS has a volume percentage of 1- $p$. Fig. 1 shows the $I-V$ characteristics of SCLDs comprising pristine $\mathrm{P} 3 \mathrm{HT}(p=1)$ and a-PS:P3HT blends with $p$ $=0.79$ and $p=0.37$. For high P3HT content in the blend $(p=$ 0.79 ) the electrical properties of the blended device are almost identical to the pristine P3HT devices. However, a significant drop in conductivity was observed when a-PS was further increased to produce the $p=0.37$ blend.

AFM and c-AFM measurements were carried out on the same films to examine the effect of blending on both surface morphology and the heterogeneity of current flow. AFM images in Fig. 2 show that the surface morphology of the P3HT and $p=$ 0.37 a-PS:P3HT blend is largely smooth. RMS roughness values for all reported SCLDs are shown in Table S1 of the ESI. $\dagger$ Similarly homogeneous current flow is shown in c-AFM images for both films, although the conductivity of the P3HT film is larger by an order of magnitude than that for the $p=0.37$ aPS:P3HT blend; the current distribution profile is presented in Fig. 3. The isotropic nature of the a-PS blends are a consequence of the atactic nature of the insulating polymer, with the

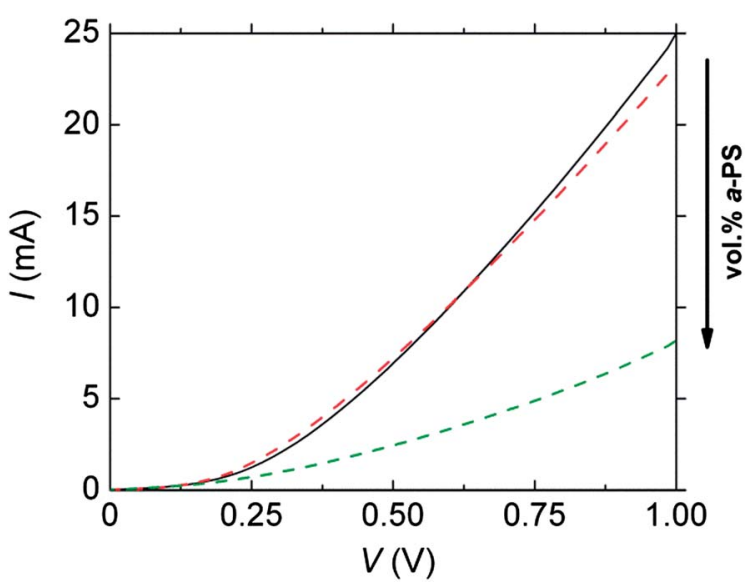

Fig. 1 Current-voltage $(I-V)$ characteristics of a pristine P3HT film (black solid), and blends of a-PS:P3HT with $p=0.79$ (red dash) and $p=$ 0.37 (green dash).
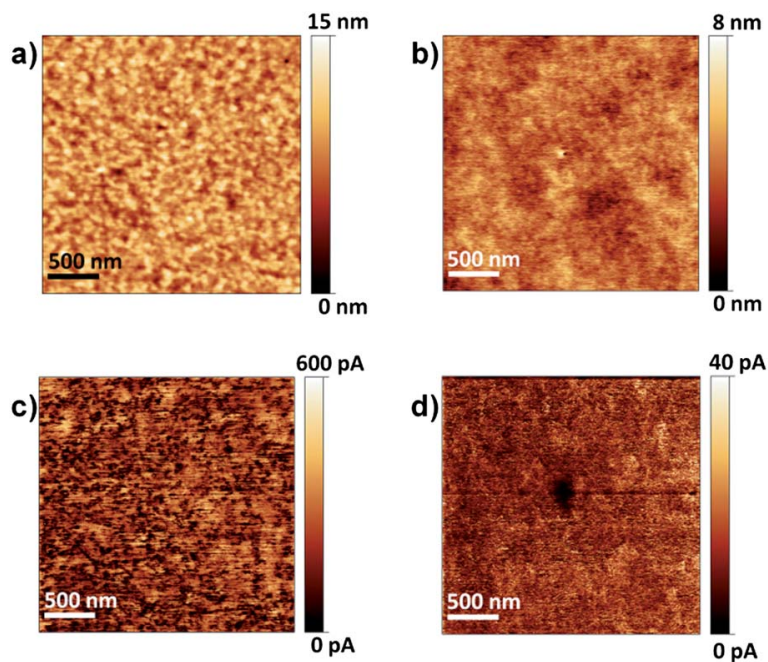

Fig. 2 AFM height images: (a) P3HT $(p=1)$ and (b) $p=0.37$ a-PS:P3HT. c-AFM images: (c) P3HT ( $p=1)$ and (d) $p=0.37$ a-PS:P3HT. AFM and cAFM data measured simultaneously. Current measured under a forward bias of $1 \mathrm{~V}$.

unfavorable stereochemistry of the phenyl groups resulting in an amorphous morphology. These findings are in agreement with results obtained from similar a-PS:P3HT OFETs, ${ }^{\mathbf{1 2}}$ where the charge carrier mobility drops approximately by an order of magnitude when P3HT content is reduced from 100 to $40 \mathrm{wt} \%$. The $1 / f$ noise characteristics for a current $I=3 \mathrm{~mA}$ are presented in Fig. 4 and reveal that the noise level is dependent on the $\mathrm{P} 3 \mathrm{HT}$ content, $p$. The $1 / f$ noise for pristine $\mathrm{P} 3 \mathrm{HT}(p=1)$ and $p=$ 0.79 a-PS:P3HT devices are shown to be of a similar magnitude, however, the noise magnitude for the $p=0.37$ a-PS:P3HT devices is significantly enhanced. This is attributed to the P3HT concentration $(p)$ approaching the percolation threshold $\left(p_{\mathrm{c}}\right){ }^{18}$ It should be noted that isolated defects can lead to variations in the $1 / f$ noise characteristics ${ }^{27}$ and hence the data presented in this paper are taken from representative devices identified from examining the distributions of a set of samples (between 5 and 25 devices).

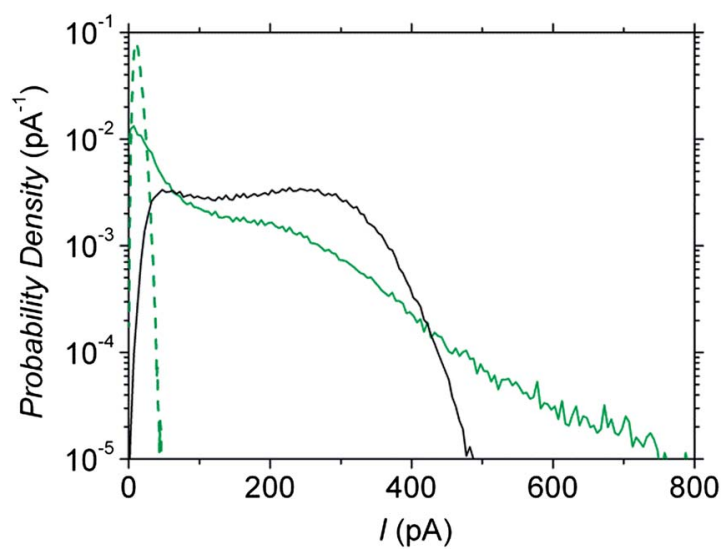

Fig. 3 c-AFM current distribution profiles for pristine P3HT $(p=1)$ (black solid), $p=0.37$ a-PS:P3HT blend (green dash) and $p=0.41$ i-PS:P3HT blend (green solid). 


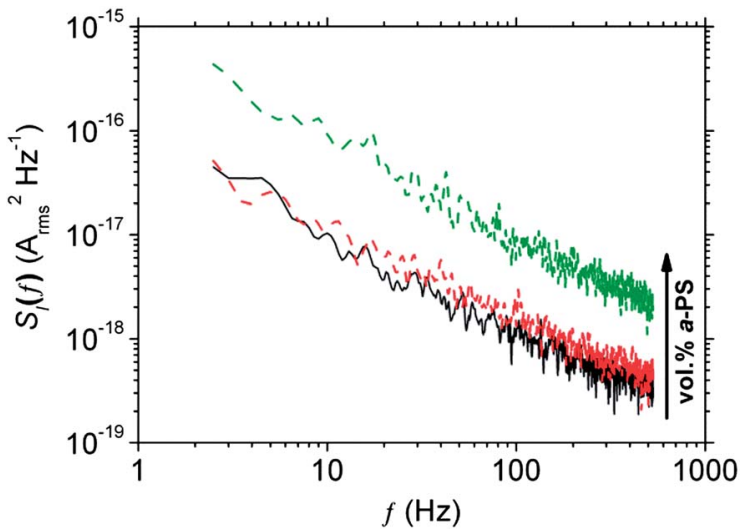

Fig. 41 /f noise spectra of pristine P3HT ( $p=1$ ) (black solid), $p=0.79$ (red dash) and $p=0.37$ (green dash) a-PS:P3HT blends.

It is important to note that our aim was to examine the conduction networks in situ, where noise sources from both the bulk and surface can apply. ${ }^{17}$ The effects of surface noise can be eliminated experimentally, ${ }^{27}$ although recent work on addressing the dominance of bulk versus surface origin of $1 / f$ noise suggests that the bulk or volume noise becomes dominant when the thickness exceeds $\sim 2.5 \mathrm{~nm} .{ }^{32}$ However, in this case the relative contribution of the bulk and the surface to the measured noise is expected to be similar for all devices measured since the active layer in all cases is $\sim 40 \mathrm{~nm}$.

\section{P3HT:i-PS blend characteristics: anisotropic networks}

Anisotropic networks were studied via the fabrication of ITO/ PEDOT:PSS/i-PS:P3HT/Au devices. Isotactic polystyrene solidifies to form an insulating semi-crystalline matrix, and it has been shown that the addition of i-PS to blends incorporating P3HT significantly increases the crystallinity of the P3HT domains, promoting the formation of vertically stratified microstructures throughout the film., ${ }^{\mathbf{6} 12}$ UV-Visible spectroscopy (Fig. S1, ESI $\dagger$ ) confirms the more crystalline nature of the P3HT domains within i-PS bends compared to those of the a-PS blends, with greater absorption observed for the $p=0.41$ i-PS:P3HT devices at approximately $570 \mathrm{~nm}$ resulting in a pronounced shoulder which signifies enhanced interchain $\pi-\pi *$ stacking. ${ }^{33-35}$

The resulting anisotropic networks are expected to be beneficial to efficient charge transport. This was observed in our devices, as depicted in Fig. 5, where it is shown that the conductivity of the $p=0.41$ i-PS:P3HT blend is significantly greater than that of the $p=0.37$ a-PS:P3HT blend. Indeed, the conductivity of the $p=0.41 \mathrm{i}-\mathrm{PS}: \mathrm{P} 3 \mathrm{HT}$ is similar, and at low bias better, than the P3HT diode. We attribute this to the observation elsewhere ${ }^{12}$ that the addition of i-PS to P3HT can improve crystallinity of the $\mathrm{P} 3 \mathrm{HT}$, and therefore charge transport, through the P3HT sections of the device. However, the improvement in charge transport with i-PS loading does not increase without bound, as higher loadings restrict the P3HT network and the current subsequently drops.

AFM and c-AFM images presented in Fig. 6 reveal the heterogeneous nature of the surface morphology, and it can be

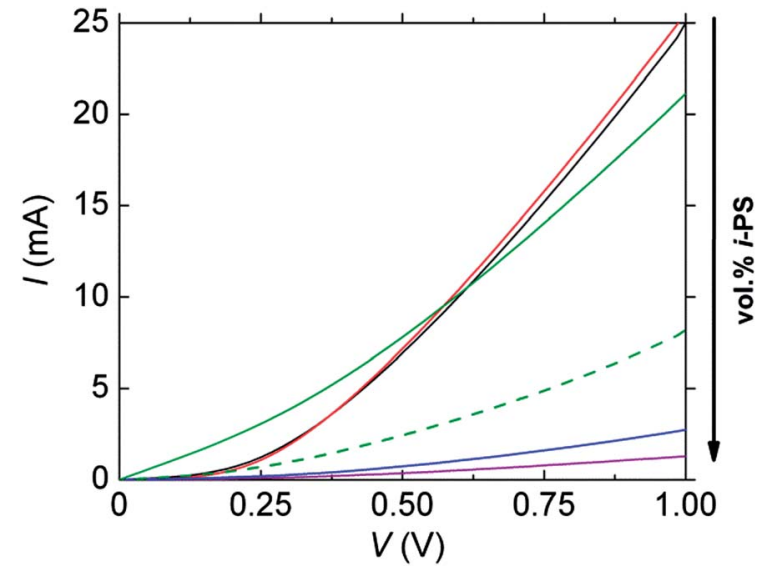

Fig. $5 \quad I-V$ characteristics of pristine P3HT ( $p=1)$ (black solid), $p=0.37$ a-PS:P3HT blend (green dash) and the following i-PS:P3HT blends (solid): $p=0.80$ (red), $p=0.41$ (green), $p=0.21$ (blue) and $p=0.12$ (purple).

seen that hole transport occurs through 'hotspots' of recessed P3HT-rich domains. We note, however, that conduction can be seen through minority P3HT within the i-PS-rich domains. This behavior is in contrast to the more homogenous morphology and conduction of the $p=0.37$ a-PS:P3HT blend in Fig. 2 . As the concentration of i-PS increases still further, the size of P3HT domains reduces which results in a concomitant reduction in the current with heterogeneous charge transport being preserved. c-AFM current distribution profiles shown in Fig. 3 quantify the heterogeneity in conduction of the $p=0.41 \mathrm{i}$ PS:P3HT devices, showing that the burden of the increased conduction falls to a few small areas of the device whereas the $p$ $=0.37$ a-PS:P3HT blends display more symmetrical distribution profiles, suggestive of more homogenous (albeit lower) conduction. ${ }^{36}$
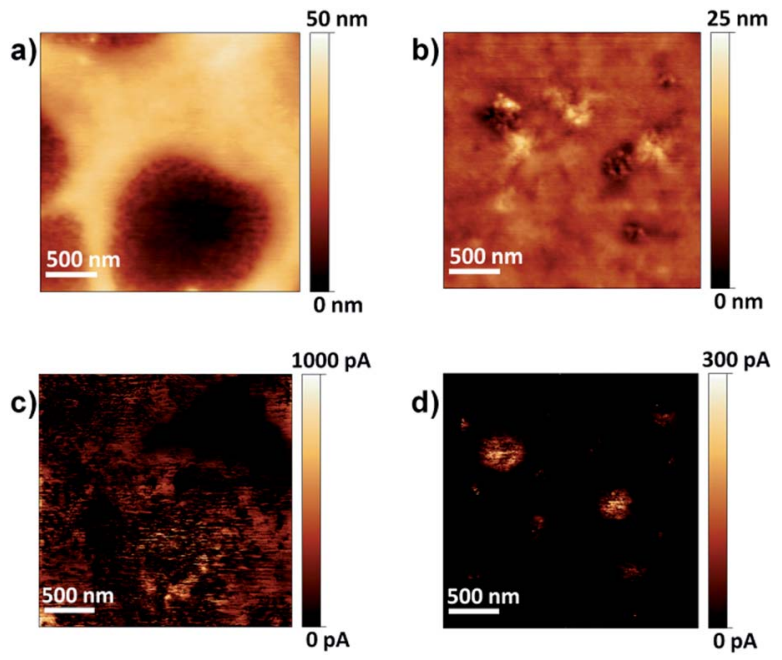

Fig. 6 AFM height images: (a) $p=0.41$ i-PS:P3HT and (b) $p=0.21$ i-PS:P3HT. C-AFM images: (c) $p=0.41$ i-PS:P3HT and (d) $p=0.21$ i-PS:P3HT. AFM and C-AFM data measured simultaneously. Current measured under a forward bias of $1 \mathrm{~V}$. 
We note that the concentration of P3HT within P3HT:i-PS blends at which point the conductivity begins to drop is below a concentration of $0.10 \mathrm{wt} \%$ for OFETs, ${ }^{12}$ where charge transport is in-plane, and below $p \sim 0.40$ for the present SCLDs, where charge transport is perpendicular to the substrate. We attribute this difference to possible vertical stratification of P3HT and i-PS in these blended devices. ${ }^{12}$

The $1 / f$ noise characteristics for i-PS blends at a bias current of $3 \mathrm{~mA}$, presented in Fig. 7, show the dependency of the noise magnitude on $p$. When comparing the $1 / f$ spectra for the approximately $p=0.4$ PS:P3HT blends, the a-PS blend has a significantly enhanced noise magnitude, lying approximately between the noise levels of the $p=0.21$ and $p=0.12$ i-PS:P3HT blends. We attribute this difference to the different values of $p_{\mathrm{c}}$ for the two blends, leading to different scaling of the noise level as per eqn (1). We additionally note that the noise for the $p=$ 0.80 i-PS:P3HT blend is slightly below that of the P3HT device. We speculate that this may be due to greater crystallisation in the $p=0.80$ i-PS:P3HT than in annealed P3HT only $(p=1)$ device, as shown elsewhere. ${ }^{12}$ This in turn, we believe, means there is a greater fraction of the P3HT volume which is crystalline in the blended device, making the transport network more homogeneous, and in turn leading to lower noise.

Fig. 8 summarises the variation in current at $1 \mathrm{~V}$ for the PS:P3HT blends as a function of $p$ for both i-PS and a-PS blends. From this we estimate the i-PS:P3HT blends have $p_{\mathrm{c}} \sim 0.10$, as compared to the a-PS:P3HT blends which have $p_{\mathrm{c}} \sim 0.25$, the differences arising due to the different modes of conduction through the anisotropic i-PS and isotropic a-PS blends. The value of $p_{\mathrm{c}}$ was approximated as the percolating fraction for which the current is a factor of 20 smaller than that for equivalent P3HT $(p=1)$ diode. This required a linear extrapolation of the a-PS blend data shown in Fig. 8. By fitting $\log S_{I}(f)$ at $f=10$ $\mathrm{Hz}$ against $\log \left(p-p_{\mathrm{c}}\right)$ we obtain approximate values for $\kappa$ of $\sim 1.1$ for the i-PS blend and $\kappa \sim 1.6$ for the a-PS blend. These values are similar to theoretical values of $\kappa$ for a $2 \mathrm{D}$ and $3 \mathrm{D}$ lattice; $\kappa=1.12$ and 1.56 respectively. ${ }^{27}$ These seem to resonate with the findings of c-AFM, since conduction occurs

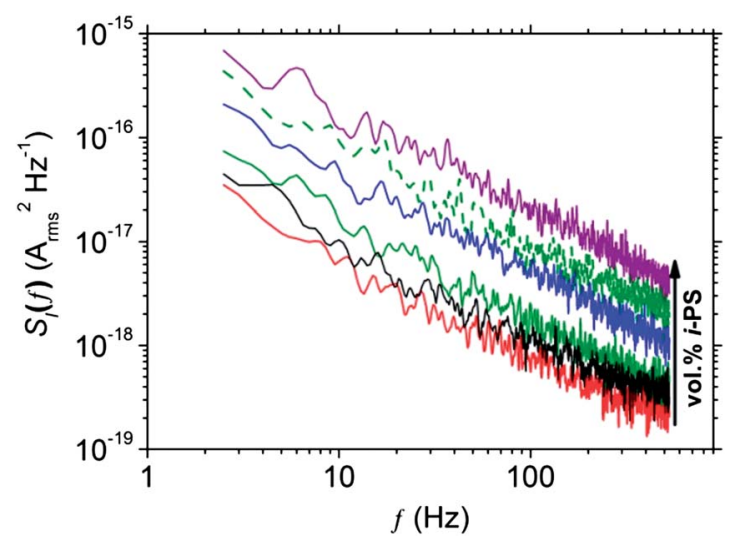

Fig. $71 / f$ noise spectra of pristine P3HT $(p=1)$ (black solid), $p=0.37$ a-PS:P3HT blend (green dash), and the following i-PS:P3HT blends (solid): $p=0.80$ (red), $p=0.41$ (green), $p=0.21$ (blue) and $p=0.12$ (purple).

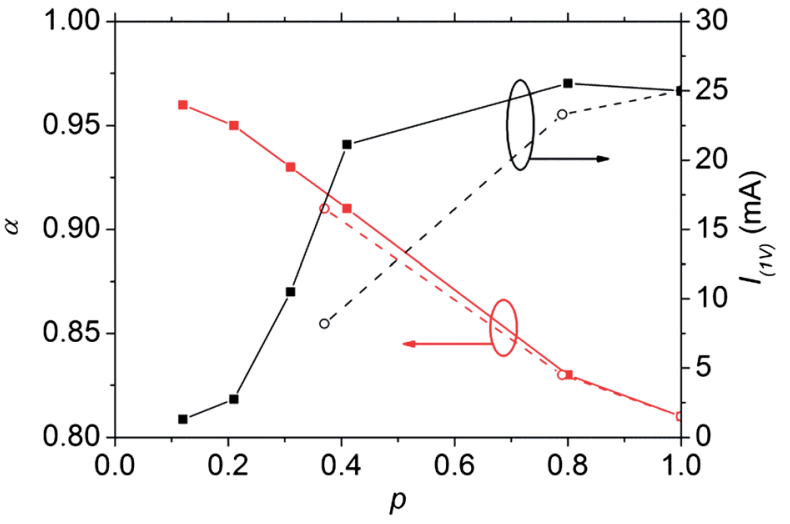

Fig. 8 The current at $1 \mathrm{~V}\left(I_{1 V}\right)$ (black) and $\alpha$ (red) plotted against volume content of P3HT, $p$. Shown are data for a-PS:P3HT (dashed, open circles) and i-PS:P3HT blends (solid, closed squares).

homogeneously throughout the a-PS blend, whereas the conduction routes through the i-PS blend are more limited. In this case, studying how noise varies with blend concentration reveals information about the nature of conduction through the active medium. Understanding how changes in network nanostructure relates to device performance is crucial in furthering the development of organic electronics, for example, the process of annealing bulk heterojunction OPVs promotes nanoscale phase separation which can improve device efficiency. Of course, such network information is available through other techniques, such as c-AFM, ${ }^{36}$ scanning transmission X-ray microscopy (STXM) ${ }^{37}$ and electron tomography. ${ }^{38}$ Our results suggest that $1 / f$ noise can be used to examine the network from the viewpoint of the charges, since the form of the network the charges travel through is revealed by $\kappa$. However, it should be noted that in OPV blends both networks are semiconducting, as opposed to just one here. The use of noise spectroscopy in OPV blends to probe morphology will be examined in further publications.

\section{1/f noise slope and the density of states}

If we consider $S_{I}(f)$ to be described by the function $1 / f^{\alpha}$, the fitted value of $\alpha$ was found to approach unity as $p$ reduced, as shown in Fig. 8. The slope of the noise can be thought of as a consequence of the dynamics of defect state trapping and detrapping events, since a single trap with a single relaxation time will contribute noise power with a Lorentzian function ${ }^{17,20}$ frequency dependence, i.e. $1 / f^{2}$. Therefore $\alpha<2$ generally indicates the presence of a multiplicity of trap states, with smaller $\alpha$ indicating a more extended density of states with an associated set of oscillators. ${ }^{\mathbf{1 6 , 2 0}}$ A broadened DoS is expected in conjugated polymers such as $\mathrm{P} 3 \mathrm{HT}$ due to the random conformation of chains throughout the film. ${ }^{39}$ Often the DoS can be described by a Gaussian distribution which has a standard deviation in the region of $50-150 \mathrm{meV}^{\mathbf{4 0}}$ We attribute the increase of $\alpha$ as $p$ reduces shown in Fig. 8 to the increasing sparseness of the charge conduction networks, as within the confines of fewer and narrower pathways it follows that charge 
carriers will encounter a less diverse distribution of trapping centres within the DoS. A broader sampling of the DoS would be expected for a more uniformly conductive film such as that of the pristine P3HT devices ( $p=1$ ). The $\alpha$ values obtained for the a-PS blends were found to be similar to those of the i-PS blends. This suggests that the sampling of the DoS is largely unaffected by the physical routes through the film.

The use of $\alpha$ as a means to probe the DoS within organic thinfilms in situ is particularly useful, as the energetic distribution of traps will affect important device parameters and processes such as carrier mobility ${ }^{\mathbf{4 1 , 4 2}}$ and recombination kinetics, ${ }^{\mathbf{4 3}}$ which in turn can limit device working efficiency. ${ }^{43}$ However, quantitatively measuring the DoS within organic thin-films is challenging, with techniques such as capacitance-frequency ${ }^{\mathbf{4 4}}$ and transient photocurrent ${ }^{43}$ (TPC) measurements being laborious in nature, with the latter technique involving careful selection of applied voltages and preferably computational modelling to confidently measure an accurate distribution. $1 / f$ noise measurement can be used as a quick and straightforward method to provide a qualitative description of the energetic distribution for organic thin-film devices.

\section{Conclusions}

We have investigated the use of $1 / f$ noise spectroscopy in percolating blends composed of the polymers P3HT and PS in various ratios. The noise magnitude for both isotropic and anisotropic conduction networks were found to increase as the conductive P3HT content was lowered toward the percolation threshold $p_{c}$. This is confirmed with the aid of AFM and c-AFM measurements to be due to the conductive networks becoming sparser with reducing $\mathrm{P} 3 \mathrm{HT}$ content. The $1 / f$ spectrum was found to be sensitive to the degree of morphological heterogeneity, with the noise magnitude being substantially enhanced in the isotropic networks for a given PS content. Additionally, values for $p_{\mathrm{c}}$ and $\kappa$ were extracted from the data and these were found to be higher for the a-PS blends. The slope of $1 / f^{\alpha}$ noise was found to approach unity as the PS content was increased for both types of conductive networks, indicating that a smaller proportion of the density of states was sampled by the charges. We conclude that noise spectroscopy can be used for in situ probing of charge conduction networks and the density of states within thin-film organic electronics, providing an uncomplicated and inexpensive means to relate the morphological and energetic landscape within devices to their working performance.

\section{Acknowledgements}

We acknowledge Dr Christopher Pearson for AFM thin-film thickness measurements, Dr Claudio Balocco for useful conversations, and to EPSRC for funding (grant reference EP/ J009938/1).

\section{Notes and references}

1 G. Yu, J. Gao, J. C. Hummelen, F. Wudl and A. J. Heeger, Science, 1995, 270, 1789-1791.
2 J. J. M. Halls, C. A. Walsh, N. C. Greenham, E. A. Marseglia, R. H. Friend, S. C. Moratti and A. B. Holmes, Nature, 1995, 376, 498-500.

3 K. T. Kamtekar, A. P. Monkman and M. R. Bryce, Adv. Mater., 2010, 22, 572-582.

4 M. C. Gather, A. Kohnen and K. Meerholz, Adv. Mater., 2011, 23, 233-248.

5 J. Moulton and P. Smith, J. Polym. Sci., Part B: Polym. Phys., 1992, 30, 871-878.

6 T. A. M. Ferenczi, C. Muller, D. D. C. Bradley, P. Smith, J. Nelson and N. Stingelin, Adv. Mater., 2011, 23, 4093-4097.

7 C. J. Brabec, M. Heeney, I. McCulloch and J. Nelson, Chem. Soc. Rev., 2011, 40, 1185-1199.

8 C. R. McNeill and N. C. Greenham, Adv. Mater., 2009, 21, 3840-3850.

9 F. Dou, C. Silva and X. Zhang, Chem. Phys. Lett., 2013, 572, 44-47.

10 N. Shin, J. Kang, L. J. Richter, V. M. Prabhu, R. J. Kline, D. A. Fischer, D. M. DeLongchamp, M. F. Toney, S. K. Satija, D. J. Gundlach, B. Purushothaman, J. E. Anthony and D. Y. Yoon, Adv. Funct. Mater., 2013, 23, 366-376.

11 B. A. Collins, E. Gann, L. Guignard, X. He, C. R. McNeill and H. Ade, J. Phys. Chem. Lett., 2010, 1, 3160-3166.

12 S. Goffri, C. Muller, N. Stingelin-Stutzmann, D. W. Breiby, C. P. Radano, J. W. Andreasen, R. Thompson, R. A. J. Janssen, M. M. Nielsen, P. Smith and H. Sirringhaus, Nat. Mater., 2006, 5, 950-956.

13 R. Giridharagopal and D. S. Ginger, J. Phys. Chem. Lett., 2010, 1, 1160-1169.

14 D. M. DeLongchamp, R. J. Kline and A. Herzing, Energy Environ. Sci., 2012, 5, 5980-5993.

15 J. Muller, ChemPhysChem, 2011, 12, 1222-1245.

16 M. Bag, N. S. Vidhyadhiraja and K. S. Narayan, Appl. Phys. Lett., 2012, 101, 043903.

17 F. N. Hooge, IEEE Trans. Electron Devices, 1994, 41, 19261935.

$18 \mathrm{~S}$. Kogan, Electronic noise and fluctuations in solids, Cambridge, 1996.

19 R. Rammal, C. Tannous, P. Breton and A. M. S. Tremblay, Phys. Rev. Lett., 1985, 54, 1718-1721.

20 A. K. Raychaudhuri, Curr. Opin. Solid State Mater. Sci., 2002, 6, 67-85.

21 O. D. Jurchescu, B. H. Hamadani, H. D. Xiong, S. K. Park, S. Subramanian, N. M. Zimmerman, J. E. Anthony, T. N. Jackson and D. J. Gundlach, Appl. Phys. Lett., 2008, 92, 132103.

22 C. Bonavolonta, C. Albonetti, M. Barra and M. Valentino, J. Appl. Phys., 2011, 110, 093716.

23 Y. Xu, T. Minari, K. Tsukagoshi, J. Chroboczek, F. Balestra and G. Ghibaudo, Solid-State Electron., 2011, 61, 106-110.

24 L. Ke, S. Bin Dolmanan, C. Vijila, S. J. Chua, Y. H. Han and T. Mei, IEEE Trans. Electron Devices, 2010, 57, 385-390.

25 H. Kang, L. Jagannathan and V. Subramanian, Appl. Phys. Lett., 2011, 99, 062106.

26 G. Ferrari, D. Natali, M. Sampietro, F. P. Wenzl, U. Scherf, C. Schmitt, R. Guntner and G. Leising, Org. Electron., 2002, $3,33-42$. 
27 J. Planes and A. Francois, Phys. Rev. B: Condens. Matter Mater. Phys., 2004, 70, 184203.

28 B. R. Conrad, W. G. Cullen, W. Yan and E. D. Williams, Appl. Phys. Lett., 2007, 91, 242110.

29 T. J. Prosa, M. J. Winokur, J. Moulton, P. Smith and A. J. Heeger, Macromolecules, 1992, 25, 4364-4372.

30 G. Cheng, Y. B. Melnichenko, G. D. Wignall, F. J. Hua, K. L. Hong and J. W. Mays, Polymer, 2007, 48, 4108-4113.

31 G. Natta, P. Corradini and I. W. Bassi, II Nuovo Cimento Series 10, 1960, 15, 68-82.

32 G. Liu, S. Rumyantsev, M. S. Shur and A. A. Balandin, Appl. Phys. Lett., 2013, 102, 093111.

33 J. H. Kim, J. H. Park, J. H. Lee, J. S. Kim, M. Sim, C. Shim and K. Cho, J. Mater. Chem., 2010, 20, 7398-7405.

34 V. Shrotriya, J. Ouyang, R. J. Tseng, G. Li and Y. Yang, Chem. Phys. Lett., 2005, 411, 138-143.

35 L. Z. Qiu, W. H. Lee, X. H. Wang, J. S. Kim, J. A. Lim, D. Kwak, S. Lee and K. Cho, Adv. Mater., 2009, 21, 1349-1353.

36 L. S. C. Pingree, O. G. Reid and D. S. Ginger, Nano Lett., 2009, 9, 2946-2952.
37 C. R. McNeill, B. Watts, L. Thomsen, W. J. Belcher, N. C. Greenham and P. C. Dastoor, Nano Lett., 2006, 6, 1202-1206.

38 B. V. Andersson, S. Masich, N. Solin and O. Inganas, J. Microsc., 2012, 247, 277-287.

39 V. Coropceanu, J. Cornil, D. A. da Silva, Y. Olivier, R. Silbey and J. L. Bredas, Chem. Rev., 2007, 107, 926-952.

40 J. C. Blakesley, H. S. Clubb and N. C. Greenham, Phys. Rev. B: Condens. Matter Mater. Phys., 2010, 81, 045210.

41 M. Caironi, M. Bird, D. Fazzi, Z. H. Chen, R. Di Pietro, C. Newman, A. Facchetti and H. Sirringhaus, Adv. Funct. Mater., 2011, 21, 3371-3381.

42 P. E. Parris, D. H. Dunlap and V. M. Kenkre, Phys. Status Solidi B, 2000, 218, 47-53.

43 R. C. I. MacKenzie, C. G. Shuttle, G. F. Dibb, N. Treat, E. von Hauff, M. J. Robb, C. J. Hawker, M. L. Chabinyc and J. Nelson, J. Phys. Chem. C, 2013, 117, 12407-12414.

44 B. Ecker, J. C. Nolasco, J. Pallares, L. F. Marsal, J. Posdorfer, J. Parisi and E. von Hauff, Adv. Funct. Mater., 2011, 21, 27052711. 